\title{
CAMA
}

Centre for Applied Macroeconomic Analysis

\section{Social distancing and contagion in a discrete choice model of COVID-19}

\section{CAMA Working Paper 35/2020 April 2020}

\section{Giorgos Baskozos}

University of Oxford, UK

\section{Giorgos Galanis}

Goldsmiths, University of London, UK

Centre for Applied Macroeconomic Analysis, ANU

\section{Corrado Di Guilmi}

University of Technology Sydney, Australia

Centre for Applied Macroeconomic Analysis, ANU

\section{Abstract}

We present an epidemic model in which heterogenous agents choose whether to enact social distancing practices. The policy maker decides on the timing and the extent of policies that incentivise social distancing. We evaluate the consequences of interventions and find that: (i) the timing of intervention is paramount in slowing the contagion, and (ii) a delay cannot be compensated by stronger measures. 


\title{
Keywords
}

\author{
JEL Classification
}

\section{Address for correspondence:}

(E) cama.admin@anu.edu.au

ISSN 2206-0332

The Centre for Applied Macroeconomic Analysis in the Crawford School of Public Policy has been established to build strong links between professional macroeconomists. It provides a forum for quality macroeconomic research and discussion of policy issues between academia, government and the private sector.

The Crawford School of Public Policy is the Australian National University's public policy school, serving and influencing Australia, Asia and the Pacific through advanced policy research, graduate and executive education, and policy impact. 


\title{
Social distancing and contagion in a discrete choice model of COVID-19
}

\author{
Giorgos Baskozos* ${ }^{*}$ Giorgos Galanis ${ }^{\dagger}$ P Corrado Di Guilmiः
}

April 14, 2020

\begin{abstract}
We present an epidemic model in which heterogenous agents choose whether to enact social distancing practices. The policy maker decides on the timing and the extent of policies that incentivise social distancing. We evaluate the consequences of interventions and find that: (i) the timing of intervention is paramount in slowing the contagion, and (ii) a delay cannot be compensated by stronger measures.
\end{abstract}

\footnotetext{
*University of Oxford, UK

${ }^{\dagger}$ Goldsmiths, University of London, UK; and Centre for Applied Macroeconomic Analysis, Australian National University; and CRETA, University of Warwick, UK

$\ddagger$ University of Technology Sydney, Australia; and Centre for Applied Macroeconomic Analysis, Australian National University. Corresponding author: corrado.diguilmi@uts.edu.au
} 


\section{Introduction}

The social and economic effects of COVID-19 has led to the integration of epidemic models, like the popular SIR (Kermack and McKendrick, 1927), into economics (Atkeson, 2020; Eichenbaum et al., 2020). The vast impact of COVID-19 is due to the fact that the entire population is susceptible to contagion and no pharmaceutical treatment or vaccine is currently available. Consequently, national authorities are resorting to non-pharmaceutical treatments to slow down the spread of the infection. In discussing these measures, Anderson et al. (2020) stress two crucial points. First, given the very large reproduction number (estimated to be higher than 2) mitigation strategies are paramount and social distancing appears as the most effective and feasible. Second, in enacting social-distancing policies, individual behaviour is crucial: "Personal, rather than government action, in western democracies might be the most important issue."

We present an epidemic model in which the spread of an outbreak can be mitigated by social-distancing practices, which depend on individual choices as in Eichenbaum et al. (2020). However, in consideration of the high level of uncertainty generated by the pandemic and its rapid evolution, our contribution differs by assuming that individual decisions are based on a imperfect information set, which is limited to the data about confirmed cases. We model the decision about social distancing as a binary choice drawing on the discrete choice literature (McFadden, 2001; Train, 2009) and its applications to dynamic models (Brock and Hommes, 1997; Lux, 1995, among others). The model can provide a qualitative preliminary analysis of (i) the consequences of late intervention and (ii) the effectiveness of increasing the severity of measures to compensate for the delay.

\section{Model}

Consider a large population of agents $N$, in a closed space, who face a choice between social distancing $(D)$ or not $(C)$. For all $t$, let $s_{t}$ be the share of individuals who choose $D$ at time $t$ and $1-s_{t}$ the share of people who choose 
$C$.

\subsection{Contagion}

Let $h_{t}$ be the share of confirmed infected cases over total population in period $t$ and assume that it evolves according to

$$
h_{t+1}=h_{t}\left(1+\alpha-d_{t+1}\right),
$$

where $\alpha$ is the rate of growth of confirmed cases without mitigating measures and $d_{t+1} \leq \alpha \forall t$ captures the reduction of $\alpha$ due to social distancing. In order to model the evolution of $d_{t}$, we postulate that:

A1: $d_{t} \leq a$ since we are interested in the total number of cases, abstracting from recoveries and deaths (identified as removed in SIR terminology). Accordingly, $h_{t}$ cannot decrease;

A2: $d_{t+1}>d_{t}$ for $s_{t}>0$, which means that, as long as the share of socially distancing agents is positive, the rate of increase of $h_{t}$ will be decreasing over time.

A3: $d_{t+1}=d_{t}$ for $s_{t}=0$, which means that the spread can slow down only with social distancing ${ }^{1}$.

Accordingly, we define

$$
d_{t+1}=d_{t}+\beta s_{t}\left(\alpha-d_{t}\right)
$$

where $\beta \in(0,1)$ quantifies the impact of social distancing on $\alpha$. The dynamics implied by equations (1-2) provide a good fit on the Chinese data, as shown by Figure $1 .^{2}$ For the fit, we set $\alpha=0.404, \beta s_{t}=0$ until period $t=14$, and $\beta s_{t}=0.095$ from $t \geq 14$ with $s_{t}$ constant, considering that

\footnotetext{
${ }^{1}$ However, if $h_{t}>0$, we also have $s_{t}>0$, since infected individuals enact social distancing, as discussed below.

${ }^{2}$ Data source: https://github.com/CSSEGISandData/COVID-19/ tree/master/csse_covid_19_data/csse_covid_19_time_series?fbclid= IwAR20302irrZwTKNff8-3k010XRp8jSIrSZX S-Rc0v2nuLcVIDKEyKQXJ9Go
} 
measures were imposed on day 14 after the first recorded cases.

[Figure 1 here]

\subsection{Social Distancing}

All individuals with confirmed infection choose $D$, while the rest of the population choose between $D$ and $C$ with a probability $P_{t}^{D}$ such that the share of individuals who are socially distancing at $t+1$ is

$$
s_{t+1}=h_{t}+\left(1-h_{t}\right) P_{t}^{D} .
$$

Along the lines of discrete choice models (McFadden, 2001; Train, 2009), individual choice (and hence $P_{t}^{D}$ ) depends on a vector of observable factors $x_{t}$ and unobservable individual characteristics $\epsilon_{i}$ with $i=\{1, \ldots, N\}$. Since information is limited, the two factors influencing individual decisions are assumed to be the expected number of confirmed cases of the next day $\mathbb{E}\left(h_{t+1}-h_{t}\right)$ and the costs $c$ of choosing $D$, which can be affected by government policies. Hence

$$
x_{t}=\left[\begin{array}{c}
\mathbb{E}\left(h_{t+1}-h_{t}\right) \\
-c
\end{array}\right]
$$

Individual utilities are

$$
U_{t}^{i}=\gamma x_{t}+\epsilon_{i}
$$

where $\epsilon$ follows a logistic distribution and $\gamma$ is a row vector which captures the intensity of choice. Assuming that agent $i$ chooses $D$ if $U_{t}^{i}>0$, the probability $P_{t}^{D}$ of any individual choosing $D$ at $t$ is $^{3}$

$$
P_{t}^{D}=\frac{1}{1+e^{-\gamma x_{t}}}
$$

We model the expectations $\mathbb{E}\left(h_{t+1}-h_{t}\right)$ as dependent on $d_{t}$, since from (1) it follows that $h_{t+1}-h_{t}=h_{t}\left(\alpha-d_{t}\right)$. Considering that the exact value of $d_{t}$ cannot be computed because $\epsilon_{i}$ is not observable and information about

\footnotetext{
${ }^{3}$ For details, see Train (2009).
} 
individual choices are imperfect, agents use the approximation $\tilde{d}_{t}$, calculated as

$$
\tilde{d}_{t}=\delta d_{t-1}+(1-\delta) \tilde{d}_{t-1}
$$

where $\delta \in(0,1]$ is the relative importance of the actual value in the previous period.

\section{Simulations}

We estimate $\alpha=0.35$ using the average growth of $h$ in Italy and UK and $\beta=0.10$ on Chinese data, assuming a high compliance with social distancing measures $(s=0.9)$. The other parameters are calibrated as $\gamma=[44]$, $\delta=0.5 .^{4}$ The initial value of $h$ is $h_{1}=4 \times 10^{-6}$, corresponding the proportion of cases over population of the first data record in Wuhan, while $d_{1}=\tilde{d}_{1}=s_{1}=0$. We define $t_{i}$ and $c_{i}$ as the time of policy intervention and the social-distancing costs after intervention, respectively.

[Figure 2 here]

Figure 2 shows the cumulative cases (top panel) and the daily variation (bottom panel) for three different values of $c$ which correspond to no intervention $\left(c=c_{i}=0.5\right)$, mild measures $\left(c_{i}=0.45\right.$ from period $\left.t_{i}=7\right)$, and strict measures $\left(c_{i}=0.35\right.$ from period $\left.t_{i}=7\right)$. The different policies have effects not only on the new daily cases, dramatically flattening the curve as in the standard SIR models, but also on the cumulative number. The higher incentives to social distancing are successful in substantially reducing the time for the extinction of the contagion (the point where $\Delta h=0$ ). In terms of social costs, the reduction in the contagion and in the cumulative number of cases reduces the probability for the outbreak to overwhelm the health system capacity.

\footnotetext{
${ }^{4}$ The values of $\gamma$ are chosen to ensure reasonable values for $P^{D}$ : for $c=0.5$ and $h_{t}=0.5$, we have $P_{t}^{D}=0.5$ and for $h_{t}=0$, we have $P_{t}^{D}=\frac{1}{1+e^{2}} \approx 0.12$.
} 
[Figure 3 here]

Figure 3 shows the relevance of the timing of intervention for on the (long-run) steady state value of the cumulative confirmed cases $h$, for different values of $c$. The steady state value of $h$ is increasing in $t_{i}$ and decreasing in the strength of the intervention. Particularly in the initial stages of the outbreak, an early intervention has a strong impact. For example, for the same level of intervention, an implementation at day 10 rather than at day 5 more than doubles the final total number of confirmed cases.

[Figure 4 here ]

Figure 4 provides further insights on the joint effect of timing and size of policies on the long run level of $h$. The plot demonstrates that, although the extent of the measures matters, the effect of timing dominates: for any possible level of $c \in[0,0.45]$, an early intervention always delivers a lower total number of cases.

[Figure 5 here ]

Finally, Figure 5 compares the results for an early and mild intervention $\left(t_{i}=7, c_{i}=0.45\right)$ with a later and stronger one $\left(t_{i}=21, c_{i}=0.25\right)$. Besides reducing the total number of cases, the early measure anticipates and lowers the peak in their growth, considerably easing the pressure on the health system.

\section{Discussion and conclusions}

Both the COVID-19 pandemic and the policies enacted to tackle it are having vast social and economic consequences. Our model provides a basic framework for analysing the effectiveness of mitigating policies. A more precise assessment will be possible only with more available data. Nonetheless, our analysis can provide some basic policy indications and contributes in defining 
a path for future research on the topic by identifying the role of three main factors.

\subsection{Individual choices}

In applying $S I R$ models, one should consider that individuals make choices in matter of social distancing, and these decisions play a decisive role in the evolution of total number of people infected and the shape of the curve of daily confirmed cases. Consequently, individual decision mechanisms should be taken into account in formulating and evaluating policies. In particular, the delay in the effects of individual decisions should be considered when imposing or lifting restrictions.

\subsection{Time of intervention}

The timing of policy interventions significantly impacts on the cumulative number of cases, its evolution over time, and the total time required for the contagion to die out. In particular, the analysis reveals that early mild measures seem to be in general more effective than late harsher policies. Given the vast impact of the restrictions on businesses, an untimely policy intervention requiring stricter limitations may not only have a large costs in terms of human lives but also huge economic implications.

\subsection{Uncertainty}

Given the importance of individual decisions, the provision of reliable information deserves careful consideration. For example, mass-testing can provide a more accurate estimation of the actual extent of the pandemic and exert effects in two ways: (i) directly, by leading confirmed cases to self-isolate, and (ii) indirectly, by affecting the public perception of the probability of getting infected and therefore the attitude towards social distancing. 


\section{Acknowledgments}

We wish to thank Marco Faravelli, Achilleas Mantes, and Herakles Polemarchakis, for useful comments on an earlier draft of this paper.

\section{References}

Anderson, R. M., Heesterbeek, H., Klinkenberg, D. and Hollingsworth, T. D. (2020): How will country-based mitigation measures influence the course of the COVID-19 epidemic?, The Lancet, 395: pp. 931-934, doi:10.1016/S0140-6736(20)30567-5.

Atkeson, A. (2020): What Will Be the Economic Impact of COVID-19 in the US? Rough Estimates of Disease Scenarios, Working Paper 26867, National Bureau of Economic Research.

Brock, W. and Hommes, C. (1997): A Rational Route to Randomness, Econometrica, 65: pp. 1059-1095.

Eichenbaum, M. S., Rebelo, S. and Trabandt, M. (2020): The Macroeconomics of Epidemics, Working Paper 26882, National Bureau of Economic Research.

Kermack, W. O. and McKendrick, A. G. (1927): A Contribution to the Mathematical Theory of Epidemics, Proceedings of the Royal Society of London, Series A 115(772): pp. 700-721.

Lux, T. (1995): Herd Behaviour, Bubbles and Crashes, Economic Journal, 105: pp. 881-889.

McFadden, D. (2001): Economic Choices, American Economic Review, 91(3): pp. 351-378.

Train, K. (2009): Discrete Choice Methods with Simulation, Princeton: Cambridge University Press. 


\section{Figures}

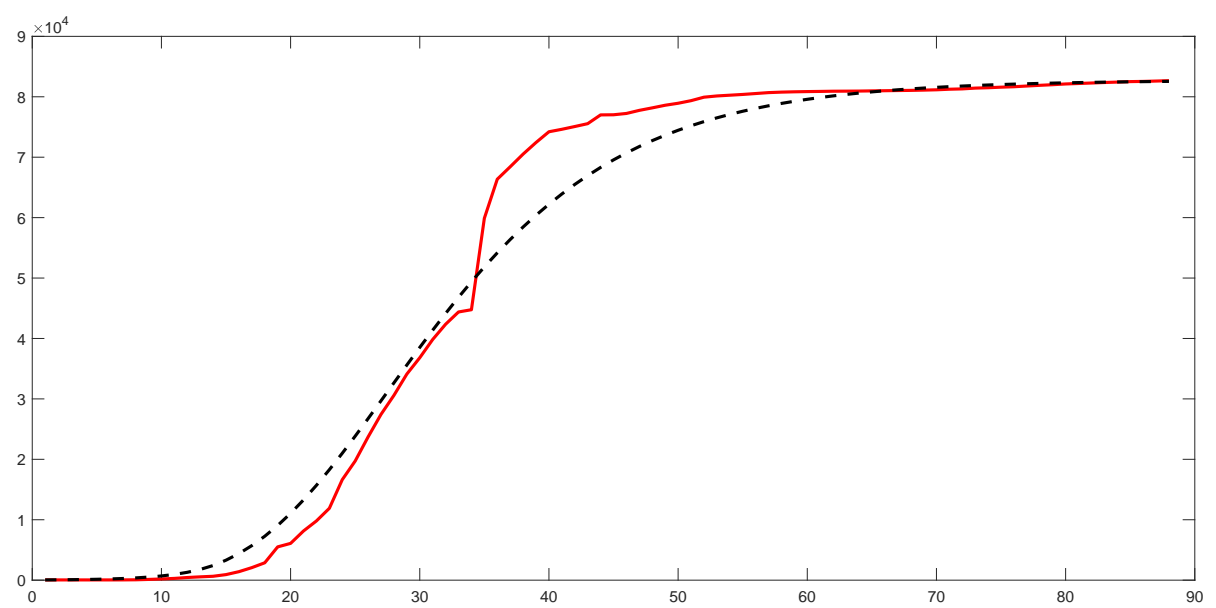

Figure 1: Number of confirmed cases, China (continuous red line) and simulations (dashed black line). 

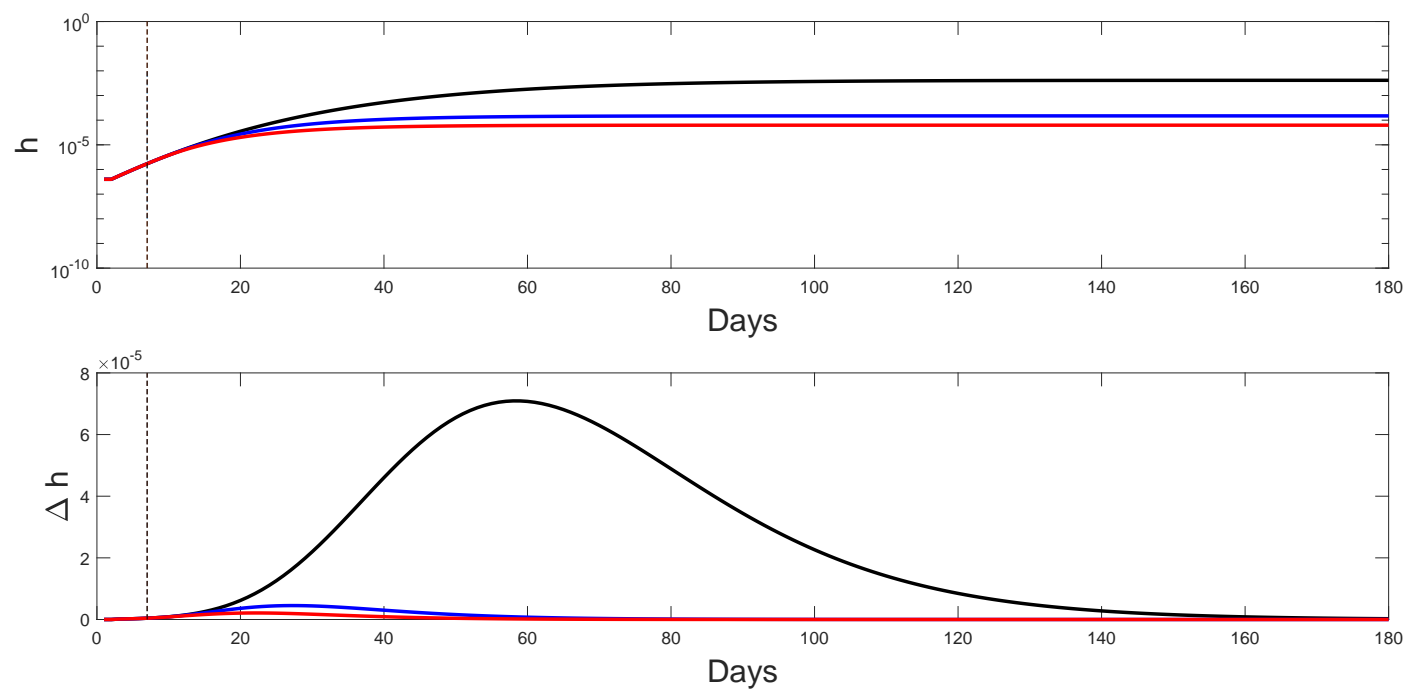

Figure 2: Simulations results with no intervention (black line), $c_{i}=0.45$ (blue line), and $c_{i} 0.35$ (red line). The dashed line marks the intervention.

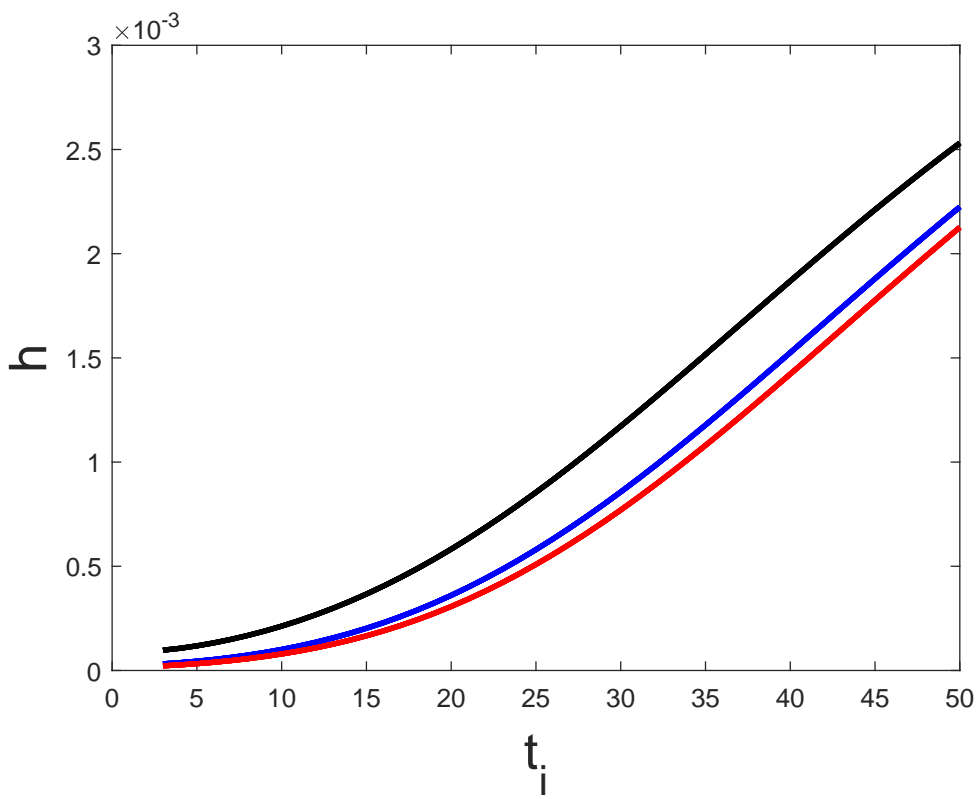

Figure 3: Steady state of $h_{t}$ as a function of $t_{i}$ for $c_{i}=0.45$ (black line), $c_{i}=0.35$ (blue line) and $c_{i}=0.25$ (red line). 


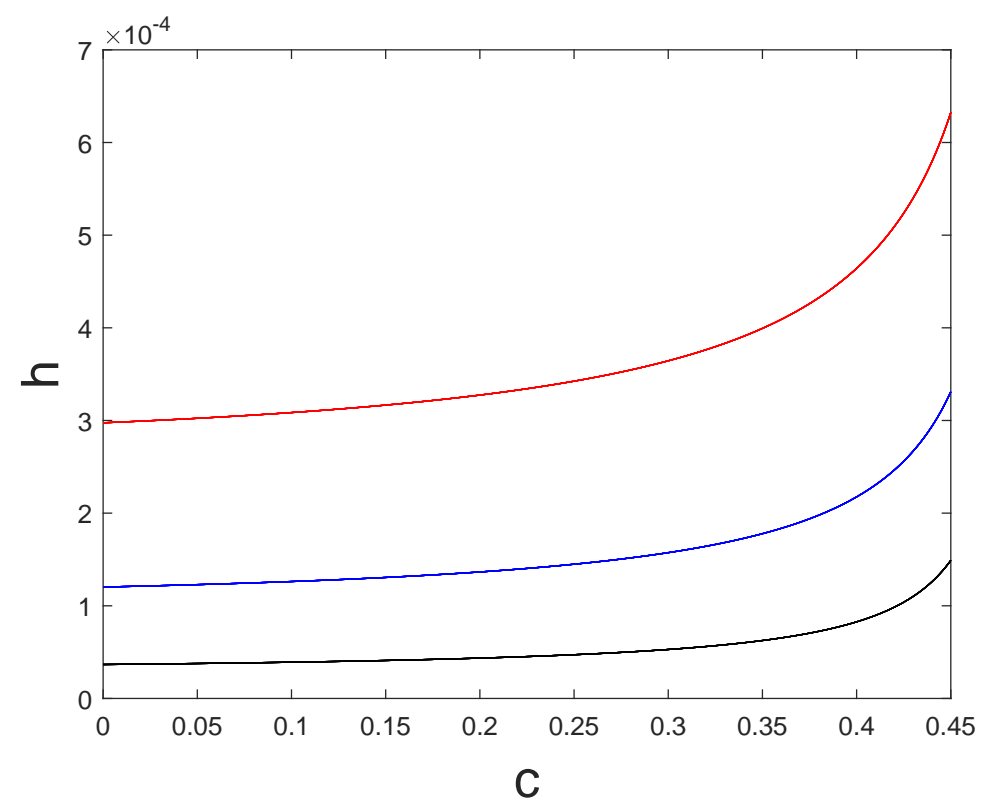

Figure 4: Steady state of $h_{t}$ as a function of $c_{i}$ for $t_{i}=7$ (black line), $t_{i}=14$ (blue line) and $t_{i}=21$ (red line).
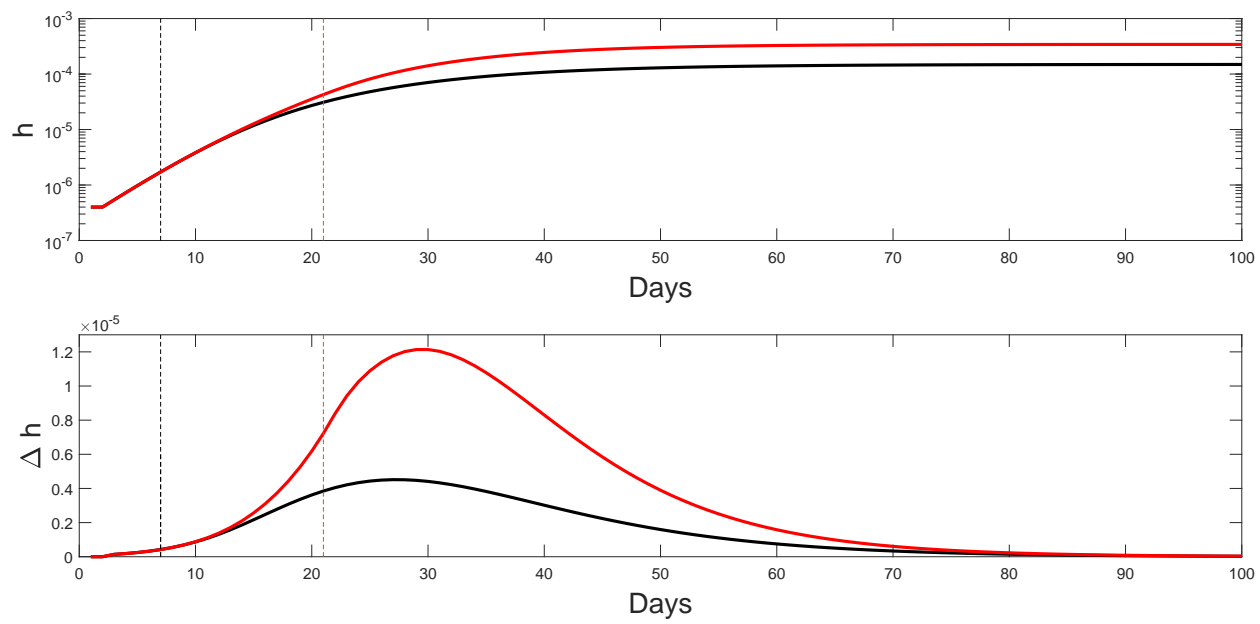

Figure 5: Simulations results for $c_{i}=0.45, t_{i}=7$ (black line) and $c_{i}=$ $0.25, t_{i}=21$ (red line). The dashed lines mark the interventions. 